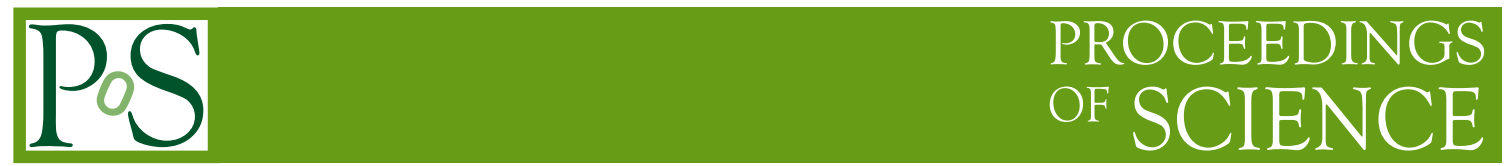

\title{
Correlation effects in multiple hard scattering
}

\section{Markus DIEHL*}

Deutsches Elektronen-Synchroton DESY, 22603 Hamburg, Germany

E-mail: markus.diehledesy.de

Correlations between the incoming partons in multiple hard scattering can affect both the event rate and kinematic distributions in the final state. In this talk, I discuss different types of correlations relevant for double parton scattering in proton-proton collisions.

XXI International Workshop on Deep-Inelastic Scattering and Related Subjects-DIS2013, 22-26 April 2013

Marseilles, France

${ }^{*}$ Speaker. 


\section{Introduction}

Multiparton interactions in proton-proton collisions, i.e. interactions in which more than one parton in one proton scatters on partons in the other proton, pose a challenge for understanding the hadronic final state at the LHC. In this talk, I concentrate on the case with two hard-scattering subprocesses in one collision, termed double parton scattering (DPS). Assuming factorization, the leading-order cross section for DPS can be written as

$$
\frac{d \sigma_{\text {double }}}{d x_{1} d \bar{x}_{1} d x_{2} d \bar{x}_{2}}=\frac{1}{C} \sum_{a_{1} a_{2} \bar{a}_{1} \bar{a}_{2}} \hat{\sigma}_{a_{1} \bar{a}_{1}} \hat{\sigma}_{a_{2} \bar{a}_{2}} \int d^{2} \mathbf{b} F_{a_{1} a_{2}}\left(x_{1}, x_{2}, \mathbf{b}\right) F_{\bar{a}_{1} \bar{a}_{2}}\left(\bar{x}_{1}, \bar{x}_{2}, \mathbf{b}\right)
$$

where $\hat{\sigma}_{a_{i} \bar{a}_{i}}$ is the cross section for the hard subprocess initiated by partons $a_{i}$ and $\bar{a}_{i}$, and $C$ is a combinatorial factor. $F_{a_{1} a_{2}}\left(x_{1}, x_{2}, \mathbf{b}\right)$ is a double parton distribution (DPD), which depends on the momentum fractions $x_{i}$ of the two partons and on their relative transverse distance $\mathbf{b}$. Higher-order corrections to $\hat{\sigma}_{a_{i} \bar{a}_{i}}$ can be incorporated into (1.1) in the same way as for single hard scattering. DPDs are essentially unknown functions, and for phenomenology one needs an ansatz for them. The simplest assumption is to write them as a product

$$
F_{a_{1} a_{2}}\left(x_{1}, x_{2}, \mathbf{b}\right)=f_{a_{1}}\left(x_{1}\right) f_{a_{2}}\left(x_{2}\right) G(\mathbf{b})
$$

of single-parton densities $f_{a}(x)$ and a factor $G(\mathbf{b})$ describing the dependence on the interparton distance. Assuming that this factor is the same for all parton types, we obtain

$$
\frac{d \sigma_{\text {double }}}{d x_{1} d \bar{x}_{1} d x_{2} d \bar{x}_{2}}=\frac{1}{C} \frac{d \sigma_{1}}{d x_{1} d \bar{x}_{1}} \frac{d \sigma_{2}}{d x_{2} d \bar{x}_{2}} \frac{1}{\sigma_{\text {eff }}}
$$

from (1.1), with a universal factor $1 / \sigma_{\text {eff }}=\int d^{2} \mathbf{b}[G(\mathbf{b})]^{2}$ and cross sections $\sigma_{i}$ for single hard scattering. This "pocket formula" provides a convenient first estimate for the size of DPS in different processes. Experimental studies at the Tevatron and the LHC yield values of $\sigma_{\text {eff }}$ between 10 and $20 \mathrm{mb}$, which are consistent with each other within their errors [1].

Let us see what follows for $\sigma_{\text {eff }}$ if there are no correlations at all in the distribution of two partons. The DPD can then be written as

$$
F_{a_{1} a_{2}}\left(x_{1}, x_{2}, \mathbf{b}\right)=\int d^{2} \mathbf{b}^{\prime} f_{a_{1}}\left(x_{1}, \mathbf{b}^{\prime}+\mathbf{b}\right) f_{a_{2}}\left(x_{2}, \mathbf{b}^{\prime}\right),
$$

where $f_{a}(x, \mathbf{b})$ is the probability density for finding a parton $a$ with momentum fraction $x$ at transverse position $\mathbf{b}$ inside the proton. Information about this distribution can be inferred from generalized parton distributions measured in exclusive scattering processes [2] and from nucleon form factors [3]. If one further assumes a universal $\mathbf{b}$ dependence for all partons, $f_{a}(x, \mathbf{b})=f_{a}(x) F(\mathbf{b})$, then the DPD factorizes as in (1.2), with

$$
G(\mathbf{b})=\int d^{2} \mathbf{b}^{\prime} F\left(\mathbf{b}^{\prime}+\mathbf{b}\right) F\left(\mathbf{b}^{\prime}\right) .
$$

For a Gaussian dependence of $F(\mathbf{b})$ with average $\left\langle\mathbf{b}^{2}\right\rangle$ one obtains

$$
\sigma_{\text {eff }}=4 \pi\left\langle\mathbf{b}^{2}\right\rangle=41 \mathrm{mb} \times \frac{\left\langle\mathbf{b}^{2}\right\rangle}{(0.57 \mathrm{fm})^{2}} .
$$


If $F(\mathbf{b})$ is the Fourier transform of a dipole $1 /\left(1+\Delta^{2} / M^{2}\right)^{2}$ in transverse momentum space, then there is an additional factor of $7 / 8$ on the r.h.s., so that the prefactor reads $36 \mathrm{mb}$ instead of $41 \mathrm{mb}$. Studies of generalized parton distributions give a typical range $\left\langle\mathbf{b}^{2}\right\rangle \sim(0.57 \mathrm{fm}-0.67 \mathrm{fm})^{2}$. The resulting values of $\sigma_{\text {eff }}$ are hence significantly larger than those extracted from DPS processes. This mismatch has been noticed long ago [4, 5], and different possible reasons have been put forward. Quite obviously, correlations in the distribution of two partons [4 - 14] could invalidate the estimate based on (1.4). Such correlations can be of quite different type, and in the following we will discuss some of them in more detail.

Notice that there could be correlations between two partons that invalidate the form (1.4) but still preserve factorization of the type (1.2). The pocket formula (1.3) would then hold, but $\sigma_{\text {eff }}$ could no longer be calculated from the one-particle distribution $f_{a}(x, \mathbf{b})$. We will, however, see in the following that it is plausible to expect correlations that invalidate (1.2). In this case, sufficiently precise extractions of $\sigma_{\text {eff }}$ based on (1.3) will find a dependence of this quantity on the process or on kinematics.

\section{Correlations between $x_{1}$ and $x_{2}$}

It is clear that the form (1.4) cannot hold when $x_{1}$ or $x_{2}$ becomes large, if only because DPDs should vanish at the kinematic boundary $x_{1}+x_{2}=1$. To alleviate this problem, a modified ansatz $F_{a_{1} a_{2}}\left(x_{1}, x_{2}, \mathbf{b}\right)=f_{a_{1}}\left(x_{1}\right) f_{a_{2}}\left(x_{2}\right)\left(1-x_{1}-x_{2}\right)^{n} G(\mathbf{b})$ with some power $n$ is sometimes used to suppress the region of large $x_{1}+x_{2}$. Significant correlations between the $x_{1}$ and $x_{2}$ dependence of $F\left(x_{1}, x_{2}, \mathbf{b}\right)$ have been found in a recent calculation using a constituent quark model [15], which should give a first idea on the behavior of DPDs for $x_{1}$ and $x_{2}$ above, say, 0.1 .

Both considerations do, however, leave open the possibility that correlations between the momentum fractions of the two partons are weak at small $x_{1}$ and $x_{2}$, which is the kinematic region relevant for most processes at the LHC.

\section{Correlations between $x_{1}, x_{2}$ and $\mathbf{b}$}

Studies of hard exclusive processes and of the nucleon form factors have taught us about generalized parton distributions and thus, with some degree of model dependence, about the impact parameter distribution $f_{a}(x, \mathbf{b})$ of a single parton inside the proton. In particular, measurements of $\gamma p \rightarrow J / \Psi p$ at HERA [16, 17] indicate a weak logarithmic dependence $\left\langle\mathbf{b}^{2}\right\rangle=\mathrm{const}+4 \alpha^{\prime} \log (1 / x)$ with $\alpha^{\prime} \approx 0.15 \mathrm{GeV}^{-2}=(0.08 \mathrm{fm})^{2}$ for gluons with $x$ around $10^{-3}$. Studies of nucleon form factors [3] and calculations of Mellin moments $\int d x x^{n} f_{a}(x, \mathbf{b})$ with $n=0,1,2$ in lattice QCD [18] indicate that for $x$ above 0.1 the decrease of $\left\langle\mathbf{b}^{2}\right\rangle$ with $x$ is even stronger. Future measurements at JLab, COMPASS and (hopefully one day) at EIC and $\mathrm{LHeC}$ will yield a detailed quantitative picture of the one-particle distributions $f_{a}(x, \mathbf{b})$ in the nucleon.

Given that the $\mathbf{b}$ distribution of a single parton becomes more narrow with increasing $x$, it is plausible to assume a similar correlation between the $\mathbf{b}$ dependence and $x_{1}, x_{2}$ in double parton distributions, even if the ansatz (1.4) of independent partons does not hold. As pointed out in [5], this has important consequences for multiparton interactions. The production of a particles with high transverse momentum or mass requires relatively large momentum fractions of the partons 
entering the corresponding hard interaction. This favors smaller values of $\mathbf{b}$ and thus a higher transverse overlap between the colliding protons, which in turn favors further interactions. An study with Pythia 8 has shown that such correlations have a quantitative impact for instance on the underlying event activity in $Z$ production [19]. Further investigation along these lines would be very interesting.

\section{Spin correlations}

Even in an unpolarized proton, the polarizations of two quarks or gluons can be correlated with each other. As was already noted in [20], such correlations have measurable consequences for DPS processes. Spin correlations between the incoming partons can be encoded in polarized DPDs, which need to be included in the factorization formula (1.1). If they are sizeable, these extra terms will invalidate the derivation leading to the pocket formula (1.3).

A full classification of polarized double parton distributions for quarks and gluons has been given in [21, 22], and in [22] it was shown that their size is limited by positivity bounds similar to the Soffer bound [23] for polarized single-parton densities. The implications of parton spin correlations have been investigated in [24] for the production of two electroweak gauge bosons $\left(\gamma^{*}, Z, W\right)$ followed by their decay into leptons. One finds that longitudinal quark spin correlations affect both the rate of DPS and the rapidity distribution of the decay leptons. By contrast, transverse quark spin correlations lead to a modulation in the azimuthal angle between the lepton decay planes of the two bosons. This makes it most evident that the two hard-scattering processes in DPS are not completely independent if there are correlations between the incoming partons.

A study in the MIT bag model found very significant spin correlations in DPDs [25], and one can expect a similar trend in any model that describes the proton in terms of three quarks. The perturbative splitting of a single parton into two, which describes the behavior of DPDs at small interparton distance b, also generates strong spin correlations [21]. How important spin correlations are at small $x_{i}$ and large $\mathbf{b}$ remains currently unknown.

\section{Color correlations}

Not only the spin but also the color of two partons can be correlated, and as a consequence, further terms with DPDs describing color correlations need to be added in the factorization formula (1.1). In the familiar collinear factorization formalism, such color correlations are suppressed by Sudakov factors [26, 27]. The amount of suppression depends on the hard scale of the process, and an estimate in [27] finds a suppression factor that is very small for $Q=100 \mathrm{GeV}$, but only equal to 0.5 for $Q=10 \mathrm{GeV}$.

For small measured transverse momenta, the discussion of Sudakov factors is more involved. If the produced particles are color neutral, one can extend the Collins-Soper-Sterman analysis of single Drell-Yan production [28] to DPS as shown in [21]. In this context, color correlation effects become relevant beyond the leading double logarithmic accuracy. 


\section{Conclusions}

A variety of correlations between two partons can affect the overall rate of double parton scattering processes, their kinematic dependence, and differential distributions in the final state. Moreover, such correlations are a characteristic feature of proton structure and thus of interest in their own right. There are indirect hints for correlations of non-negligible size, but there is no unambiguous evidence for far. Further theoretical and experimental studies in this area are definitely needed.

\section{References}

[1] M. Diehl, Multiparton interactions: Theory and experimental findings, these proceedings.

[2] F. Sabatié, From hard exclusive meson electroproduction to deeply virtual Compton scattering, these proceedings.

[3] M. Diehl, From form factors to generalized parton distributions, these proceedings.

[4] G. Calucci and D. Treleani, Mini-jets and the two-body parton correlation, Phys. Rev. D 57 (1998) 503 [hep-ph/9707389].

[5] L. Frankfurt, M. Strikman and C. Weiss, Dijet production as a centrality trigger for pp collisions at CERN LHC, Phys. Rev. D 69 (2004) 114010 [hep-ph/0311231].

[6] G. Calucci and D. Treleani, Proton structure in transverse space and the effective cross-section, Phys. Rev. D 60 (1999) $054023[\mathrm{hep}-\mathrm{ph} / 9902479]$.

[7] G. Calucci and D. Treleani, Multi-parton correlations and 'exclusive' cross sections, Phys. Rev. D 79 (2009) 074013 [arXiv:0901.3089].

[8] G. Calucci and D. Treleani, Disentangling correlations in multiple parton interactions, Phys. Rev. D 83 (2011) 016012 [arXiv: 1009 . 5881].

[9] L. Frankfurt, M. Strikman and C. Weiss, $3 D$ parton imaging of the nucleon in high-energy $p p$ and $p A$ collisions, Annalen Phys. 13 (2004) 665 [hep-ph/ 0410307$].$

[10] T. C. Rogers and M. Strikman, Multiple hard partonic collisions with correlations in proton-proton scattering, Phys. Rev. D 81 (2010) 016013 [arXiv: 0908 .0251].

[11] S. Domdey, H. J. Pirner and U. A. Wiedemann, Testing the scale dependence of the scale factor in double dijet production at the LHC, Eur. Phys. J. C 65 (2010) 153 [arXiv: 0906.4335 ].

[12] C. Flensburg, G. Gustafson, L. Lönnblad and A. Ster, Correlations in double parton distributions at small x, JHEP 1106 (2011) 066 [arXiv: 1103.4320].

[13] B. Blok, Yu. Dokshitzer, L. Frankfurt and M. Strikman, Origins of parton correlations in nucleon and multi-parton collisions, arXiv:1206.5594.

[14] B. Blok, Yu. Dokshitzer, L. Frankfurt and M. Strikman, Perturbative QCD correlations in multi-parton collisions, arXiv:1306.3763.

[15] M. Rinaldi, S. Scopetta and V. Vento, Double parton correlations in constituent quark models, arXiv: 1302.6462

[16] S. Chekanov et al. [ZEUS Collaboration], Exclusive photoproduction of $J / \Psi$ mesons at HERA, Eur. Phys. J. C 24 (2002) 345 [hep-ex/ 0201043 ]. 


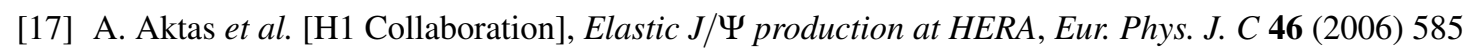
[hep-ex/0510016].

[18] Ph. Hägler, Hadron structure from lattice quantum chromodynamics, Phys. Rept. 490 (2010) 49 [arXiv:0912.5483].

[19] R. Corke and T. Sjöstrand, Multiparton interactions with an x-dependent proton size, JHEP 1105 (2011) 009 [arXiv: 1101.5953$].$

[20] M. Mekhfi, Correlations in color and spin in multiparton processes, Phys. Rev. D 32 (1985) 2380.

[21] M. Diehl, D. Ostermeier and A. Schäfer, Elements of a theory for multiparton interactions in QCD, JHEP 1203 (2012) 089 [arXiv: 1111.0910 ].

[22] M. Diehl and T. Kasemets, Positivity bounds on double parton distributions, JHEP 1305 (2013) 150 [arXiv:1303.0842].

[23] J. Soffer, Positivity constraints for spin dependent parton distributions, Phys. Rev. Lett. 74 (1995) 1292 [hep-ph/9409254].

[24] T. Kasemets and M. Diehl, Angular correlations in the double Drell-Yan process, JHEP 1301 (2013) $121[\operatorname{arXiv}: 1210.5434]$.

[25] H. -M. Chang, A. V. Manohar and W. J. Waalewijn, Double parton correlations in the bag model, Phys. Rev. D 87 (2013) 034009 [arXiv: 1211.3132 ].

[26] M. Mekhfi and X. Artru, Sudakov suppression of color correlations in multiparton scattering, Phys. Rev. D 37 (1988) 2618.

[27] A. V. Manohar and W. J. Waalewijn, A QCD analysis of double parton scattering: color correlations, interference effects and evolution, Phys. Rev. D 85 (2012) 114009 [arXiv : 1202 . 3794].

[28] J. C. Collins, D. E. Soper and G. F. Sterman, Transverse momentum distribution in Drell-Yan pair and W and Z boson production, Nucl. Phys. B 250 (1985) 199. 